\title{
Design State Feedback Controller in Multisim Software For DC - DC Buck Converter
}

\author{
Thi Ngoc Anh Dang, Viet Nguyen Hoang
}

\begin{abstract}
DC-DC convertion process is based on the principle of controlling electronic components working in linear mode (continuous converter) or switching mode (pulse converter). Later converters tend to develop due to their size and performance advantages. In this paper we will design controller for DC-DC Buck converter by state feedback control. Simulation results on the Multisim software will show the efficiency of this controller.
\end{abstract}

Index Terms-DC-DC, buck converter, multisim

\section{INTRODUCTION}

Based on the working principle, the DC-DC converter is divided into two types: continuous mode converter and switching mode converter. Continuous mode converters have the advantage of voltage quality, but electronic components working in saturation region (mosfet) or active region (BJT) lead much more consumed power. This is reason why the converters have low efficiency. Beside, in the pulse converters control, electronic components work in switching mode so the energy consumed is smaller but high efficiency.

According to [1], [2], DC-DC converters in switching mode can be classified into: Buck converter, Boot converter and Buck-boost converter. The above converters have different structure diagrams but they are based on the principle of cut - off control for semiconductor components to adjust the output voltage.

Controllers based on the output feedback structure [2], eg PID controller, have the disadvantage of slow response when some parameters of system change. For the purpose of improving the quality of the system in terms of response time, the authors use the state feedback controller applied to the buck converter.

The paper is organized follow, introduction is presented in section I, basic configuration as well as modelling the buck converter is done in section II. Section III is to propose structure controller, some calculations and simulations in multisim software are presented in section IV and conclution is in the end.

\section{CONFIGURATION OF BUCK CONVERTER}

2.1 CIRCUIT

The circuit diagram of the Buck converter is as figure 1 follows:

Thi Ngoc Anh Dang, Department of Electronics Engineering, Thai Nguyen University of Technology, Thai Nguyen, Vietnam, +84345980721

Viet Nguyen Hoang, Department of Electronics Engineering, Thai Nguyen University of Technology, Thai Nguyen, Vietnam, +84984264369

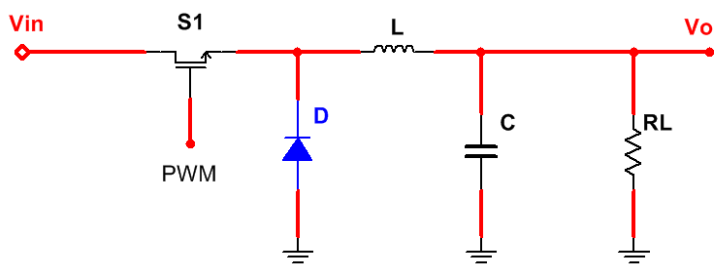

Fig 1: Circuit diagram of the Buck converter

- When transistor is on:

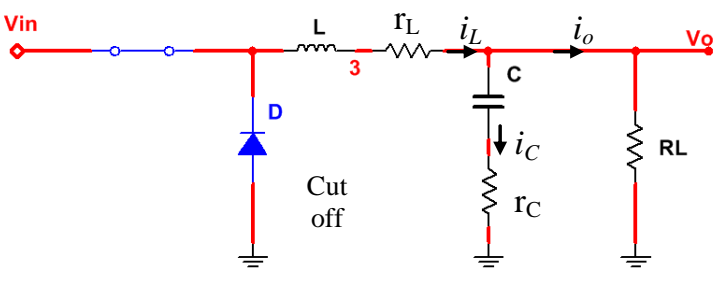

Fig 2: The circuit when trans is on

Diodes is reverse biased, $\mathrm{r}_{\mathrm{D}}=\infty$, using Kirchhoff theory, we have multi equation (1) that describe circuit

$$
\left\{\begin{array}{l}
L \frac{d i_{L}}{d t}=-r_{L} i_{L}-V_{o}+V_{i n} \\
C \frac{d u_{C}}{d t}=\frac{R}{R+r_{C}} i_{L}-\frac{1}{R+r_{C}} v_{C} \\
V_{o}=\frac{R}{R+r_{C}}\left(r_{C} i_{L}+v_{C}\right)
\end{array}\right.
$$

Put state varible $\underline{x}=\left[\begin{array}{ll}i_{L} & v_{C}\end{array}\right]^{T}$, and rewrite in state multi equations form:

$$
\left\{\begin{array}{l}
\underline{\dot{x}}=A_{1} \cdot \underline{x}+B_{1} V_{\text {in }} \\
V_{o}=C_{1} \cdot \underline{x}
\end{array}\right.
$$

We have the matrices:

$$
\begin{aligned}
& A_{1}=\left[\begin{array}{cc}
-\frac{1}{L}\left(\frac{R \cdot r_{C}}{R+r_{C}}+r_{L}\right) & -\frac{1}{L} \frac{R}{R+r_{C}} \\
-\frac{1}{C} \frac{R}{R+r_{C}} & -\frac{1}{C} \frac{1}{R+r_{C}}
\end{array}\right] ; B_{1}=\left[\begin{array}{c}
-\frac{1}{L} \\
0
\end{array}\right] ; \\
& C_{1}=\left[\begin{array}{ll}
\frac{R \cdot r_{C}}{R+r_{C}} & \frac{R \cdot r_{C}}{R+r_{C}}
\end{array}\right]
\end{aligned}
$$




\section{- When transistor is off:}

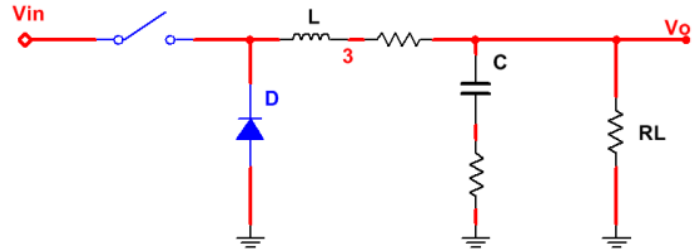

Fig 3: The circuit when trans is off

Diodes is turned on, $r_{D}=0$, we also have multi equations

(3) that describe circuit:

$$
\left\{\begin{array}{l}
L \frac{d i_{L}}{d t}=-r_{L} i_{L} \\
C \frac{d u_{C}}{d t}=\frac{R}{R+r_{C}} i_{L}-\frac{1}{R+r_{C}} v_{C} \\
V_{o}=\frac{R}{R+r_{C}}\left(r_{C} i_{L}+v_{C}\right)
\end{array}\right.
$$

Convert into state space:

$$
\left\{\begin{array}{l}
\underline{\dot{x}}=A_{2} \cdot \underline{x}+B_{2} V_{i n} \\
V_{o}=C_{2} \cdot \underline{x}
\end{array}\right.
$$

With the matrixes:

$$
\begin{aligned}
& A_{2}=\left[\begin{array}{cc}
\left(\frac{R \cdot r_{C}}{R+r_{C}}+r_{L}\right) & \frac{R}{R+r_{C}} \\
\frac{R}{R+r_{C}} & -\frac{1}{R+r_{C}}
\end{array}\right] ; B_{2}=\left[\begin{array}{c}
-\frac{1}{L} \\
0
\end{array}\right] ; \\
& C_{2}=\left[\begin{array}{ll}
\frac{R \cdot r_{C}}{R+r_{C}} & \frac{R \cdot r_{C}}{R+r_{C}}
\end{array}\right]
\end{aligned}
$$

Follow [3], model of the circuit is rewrited in mean state model:

$\left\{\begin{array}{l}\underline{\dot{x}}=\left[d A_{1}+(1-d) A_{2}\right] \cdot \underline{x}+\left[d B_{1}+(1-d) B_{2}\right] V_{i n} \\ V_{o}=\left[d C_{1}+(1-d) C_{2}\right] \cdot \underline{x}\end{array}\right.$

We have:

$$
\left\{\begin{array}{l}
{\left[\begin{array}{l}
\dot{i}_{L} \\
\dot{u}_{C}
\end{array}\right]=\left[\begin{array}{lr}
-\frac{1}{L}\left(\frac{R r_{C}}{R+r_{C}}+r_{L}\right) & -\frac{1}{L} \frac{R r_{C}}{R+r_{C}} \\
\frac{1}{C} \frac{R}{R+r_{C}} & -\frac{1}{C} \frac{R}{R+r_{C}}
\end{array}\right]\left[\begin{array}{l}
i_{L} \\
u_{C}
\end{array}\right]+\left[\begin{array}{c}
d \frac{1}{L} \\
0
\end{array}\right] V_{i n}} \\
V_{o}=\left[\begin{array}{ll}
\frac{R r_{C}}{R+r_{C}} \frac{R r_{C}}{R+r_{C}}
\end{array}\right]\left[\begin{array}{l}
i_{L} \\
u_{C}
\end{array}\right]
\end{array}\right.
$$

So the system matrixs are shown:

$$
\begin{aligned}
& A=\left[\begin{array}{cc}
-\frac{1}{L}\left(\frac{R \cdot r_{C}}{R+r_{C}}+r_{L}\right) & -\frac{1}{L} \frac{R}{R+r_{C}} \\
-\frac{1}{C} \frac{R}{R+r_{C}} & -\frac{1}{C} \frac{1}{R+r_{C}}
\end{array}\right] ; B=\left[\begin{array}{c}
-d \cdot \frac{1}{L} \\
0
\end{array}\right] ; \\
& C=\left[\begin{array}{ll}
\frac{R \cdot r_{C}}{R+r_{C}} & \frac{R \cdot r_{C}}{R+r_{C}}
\end{array}\right]
\end{aligned}
$$

\section{DESIGN CONTROLLER}

Follow the section II, after having the system matrices, we will design controller.

The structure of state feedback controller is proposed in Figure 4

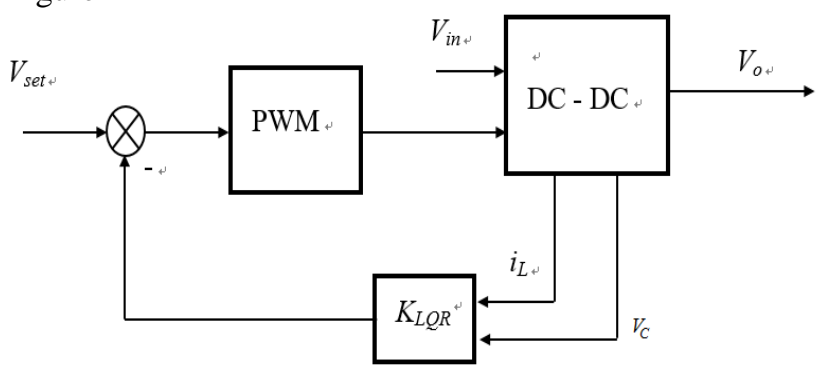

Fig 4: State feedback controller

The state feedback controller or $L Q R$ controller detemines signal control that minimizes the quadratic cost function [4]:

$$
J=\int_{0}^{\infty}\left(e^{T} Q e+u^{T} R u\right) d t
$$

where $Q$ is positive semi-definite matrix, $P$ is positive definite matrix, rule control is:

$$
K_{L Q R}=R^{-1} B^{T} P
$$

With $P$ is solution of Ricatti equation:

$$
A^{T} P+P A-P A R^{-1} B^{T} P+Q=0
$$

Finding $K$ can be done by $L Q R$ command in Matlab sofware.

\section{SOME CALCULATIONS AND SIMULATION EXPERIMENTS}

In this part, we will compute parameters and then simulate buck converter with asumptions follow:

$$
V_{\text {in }}=12 \mathrm{~V}, V_{o}=5 \mathrm{~V}, R_{L}=100 \Omega, \Delta V=100 \mathrm{~mA} \text {. }
$$

Choosing value of inductor is done by equation: [4]

$$
L=\frac{V_{o}\left(V_{i n}-V_{o}\right)}{\Delta I_{L} \cdot f_{S} \cdot V_{i n}}
$$

Where: $V_{\text {in }}$ Input voltage

$$
\begin{aligned}
& V_{o}: \text { Output voltage } \\
& f_{S} \text { : Minimum switching frequency } \\
& \Delta I_{L} \text { : Inductor ripple current, }
\end{aligned}
$$

$\Delta I_{L}$ should be chosen about from 0.2 to 0.4 output current. It means: $\Delta I_{L}=(0.2 \div 0.4) I_{\text {out } \max }$.

- Seclecting Diot: General, we choose Schottky Diot to reduce the consumer power. This Diot has to sustain the forward current and consume power:

$$
\left\{\begin{array}{l}
I_{D}=I_{\text {out }}(1-d) \\
P_{D}=I_{D} \cdot V_{D}
\end{array}\right.
$$

- For capacitor, its function is to maintain constant output voltage, so we should use the capacitor having low ESR to reduce the ripple on output voltage. Nomarly, calculating the value of capaccitor is as follow by Equation 12[4]

$$
C_{\min }=\frac{\Delta I_{L}}{8 f_{S} \Delta V_{\text {out }}}
$$

With $\Delta V_{\text {out }}$ : Peak overshoots output voltage allowed 
- Caculate the matrices:

$$
\begin{aligned}
& A=\left[\begin{array}{ll}
0 & -20000 \\
2000 & -200
\end{array}\right], B=\left[\begin{array}{c}
20000 \\
0
\end{array}\right] \\
& C=\left[\begin{array}{ll}
0 & 1
\end{array}\right]
\end{aligned}
$$

- Parametter of controller: $K=$ [2.2904 1.2306]

Simulation is implemented in Multisim software, controller is combine with: inverting amplifier Op amp, weighted summing amplifier Op amp, and shown in Figure 5

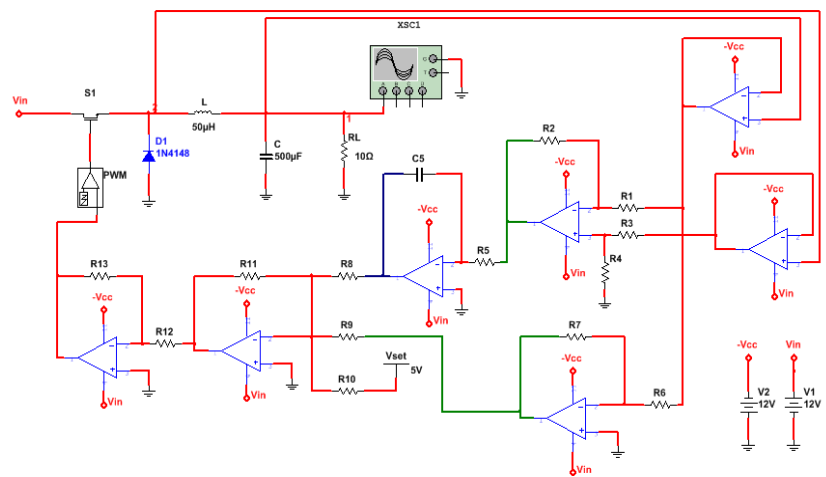

Fig 5: The circuit of DC-DC Buck converter on Multisim software

Note that, the current through the inductor is output of integrator inductor voltage by equation:

$$
i_{L}=\frac{1}{L} \int v_{L} d t
$$

The result is presented in Figure 6

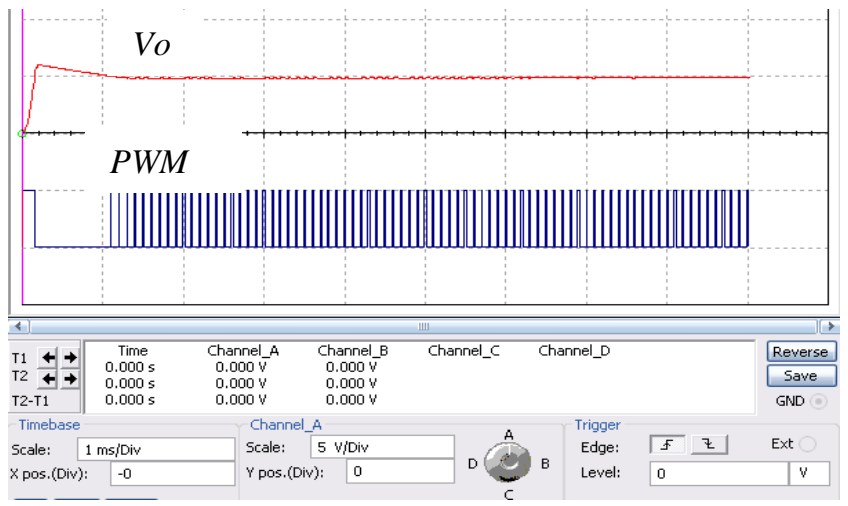

Fig 6: Output voltage of buck converter

The result show that, after $1 m s$, output voltage track the setpoint, mean value is $5 \mathrm{~V}$ with ripple voltage about $90 \mathrm{mV}$.

When we change value of load, simulation diagram and its response are shown in figure 7 and figure 8

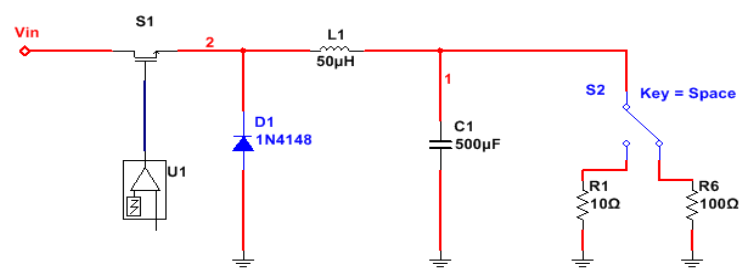

Fig 7: The circuit of DC-DC Buck converter when change value of load

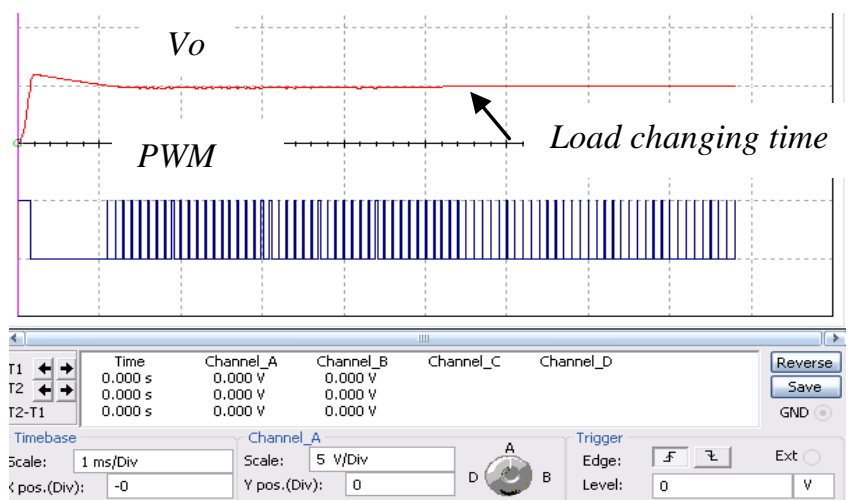

Fig 8: Output voltage when change value of load

We see that, when changing load, output still follows the setpoint, value of ripple voltage decreases (now is $22 \mathrm{mV}$ )

\section{CONCLUSION}

In this paper, we propose a state feedback controller for buck converter, the result in Multisim show that advanges of this controller is fast response time and still stable when changing load.

\section{REFERENCES}

[1] Understanding Boost Power Stages in Switch mode Power Supplies (SLVA061).

[2] Understanding Buck Power Stages in Switch mode Power Supplies (SLVA057).

[3] Robert W. Erickson, Dragan Masksimovíc (2004), Fundamentals of Power Electronic, Kluwer Academic PublishersAdel.

[4] Mousumi Mishra," Design of Buck-Boost Converter Using Multisim Software" International Journal of Engineering Research \& Technology (IJERT)

[5] Jenny Hong, Nicholas Moehle, Stephen Boyd "Linear Quadratic Control,"

[6] Linear-Quadratic Regulator (LQR) design in www.mathworks.com

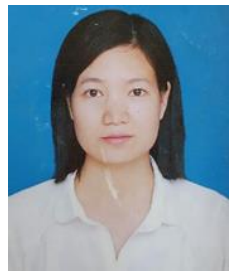

MSc Thi Ngoc Anh Dang working at Faculty of Electronics Engineering, Thai Nguyen University of Technology, Thai Nguyen city, Viet Nam. Research interests: Electronics Engineering, Automatic Control.

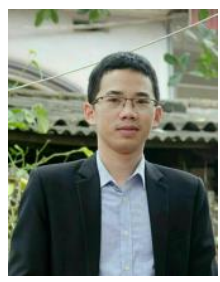

MSc Viet Nguyen Hoang working at Faculty of Electronics Engineering, Thai Nguyen University of Technology, Thai Nguyen city, Viet Nam. Now studinging at South China University or Teachnology, China. Research interests: Electronics Engineering, Automatic Control. 